\title{
Rice blast resistance gene $P$ Pii is controlled by a pair of NBS-LRR genes Pii-1 and Pii-2
}

Hiroki Takagi ${ }^{1,2}$, Akira Abe $^{1}$, Aiko Uemura ${ }^{1}$, Kaori Oikawa ${ }^{1}$, Hiroe Utsushi ${ }^{1}$, Hiroki

Yaegashi ${ }^{1}$, Hideko Kikuchi ${ }^{1}$, Motoki Shimizu, Yoshiko Abe ${ }^{1}$, Hiroyuki Kanzaki ${ }^{1}$,

Hiromasa Saito ${ }^{1}$, Ryohei Terauchi ${ }^{1,3, *}$, Koki Fujisaki ${ }^{1}$

${ }^{1}$ Iwate Biotechnology Research Center, Kitakami, Iwate, 024-0003, Japan

${ }^{2}$ Ishikawa Prefectural University, Nonoichi, Ishikawa, 921-8836, Japan

${ }^{3}$ Laboratory of Crop Evolution, Kyoto University, Muko, Kyoto 617-0001, Japan

*Author for correspondence:

Koki Fujisaki, Tel: +81-197-68-2911, E-mail: k-fujisaki@ibrc.or.jp 


\section{SUMMARY}

Nucleotide-binding, leucine-rich repeat receptors (NLRs) are conserved cytosolic receptors that recognize pathogen effectors and trigger immunity in plants. Recent studies indicate that NLRs function in pairs. Rice resistance gene Pii has been known to confer resistance against rice blast pathogen Magnaporthe oryzae carrying AVR-Pii. Previously we reported isolation of Pii gene from the rice cultivar Hitomebore (Takagi et al. 2013). To further understand rice components required for Pii-mediated resistance, we screened 5,600 mutant lines of Hitomebore cultivar and identified two mutants that lost Pii resistance without any changes in Pii gene sequence. Application of MutMap-Gap, the whole genome sequencing-based method of mutation identification, to the two mutants revealed that they have mutations in another $N L R$ gene located close to Pii. The F1 plants derived from a cross of the two mutants showed pii phenotype, demonstrating that the newly identified NLR gene is indeed a component of Pii resistance. We thus designate the previously isolated Pii gene as Pii-1 and the newly isolated NLR gene as Pii-2.

\section{Keywords}

Rice, rice blast, R-gene, NLR, whole genome sequencing, MutMap-Gap

\section{INTRODUCTION}

Plants are facing attacks from various pathogenic microbes in natural environments.

Pathogens secrete a battery of virulence effectors to manipulate host function. As a 
counter-strategy, plants evolved resistance $(R)$ genes whose products recognize pathogen avirulence effectors (AVRs). The recognition of AVRs leads to a strong defense response known as effector-triggered immunity (ETI). In most cases R proteins belong to the protein family of nucleotide-binding, leucine-rich repeat receptors (NLR) that directly or indirectly recognize AVRs (Win et al., 2012).

Mechanistic function of NLRs has not been fully understood. However, an increasing number of evidences show that NLRs cooperate with genetically linked or unlinked NLRs (Duxbury et al., 2016; Wu et al. 2017). For example, RPS4/RPS1, RGA4/RGA5 and Pikp-1/Pikp-2 are genetically linked NLR gene pairs and both of the paired NLRs are required for the recognition of cognate AVRs. One of the NLR pair is called "sensor" that recognize AVRs and the other NLR is called "helper" that is needed for the immune signaling (Duxbury et al., 2016). Genetically unlinked pairs have been also identified. Solanaceae $R$-genes $R b i$-blb2, Mi-1,2 and $R 1$ require NRC4 NLR gene in an unlinked locus and Prf R-gene requires $N R C 2$ or $N R C 3 N L R$ s (Wu et al. 2017), leading to the proposa of the concept of "NLR network" (Wu et al. 2017).

Rice blast caused by the fungal pathogen, Magnaporthe oryzae, is the most destructive disease of rice (Dean et al., 2012; Zeigler et al., 1994) and the use of $R$ genes in rice breeding is the most cost-effective measure to fight the disease (Ishizaki et al., 2005; Miah et al., 2013).

Among the cloned rice $R$-genes against M. oryzae, Pia and Pik have been reported to be composed of paired NLRs (Ashikawa et al., 2009; Okuyama et al., 2011; Yuan et al., 2011; Zhai et al., 2011). Their cognate AVRs, AVR-Pia and AVR-Pik, have also been 
isolated from M. oryzae (Yoshida et al., 2009). Pia consists of a pair of genetically linked $N L R$ genes, RGA4 and RGA5. RGA4 induces AVR-Pia-independent cell death, which is suppressed by RGA5. RGA5 directly interacts with AVR-Pia, and the RGA5-AVR-Pia interaction relieves the suppression of RGA4 function by RGA5 (Cesari et al., 2013). In this model, RGA5 functions as cell death suppressor and AVR sensor, whereas RGA4 functions as executor of defense responses. However, we still do not know whether this model proposed for Pia applies to other linked paired NLRs.

We have been studying Pii-mediated resistance of rice-M. oryzae interaction. In contrast to direct AVR recognition in Pia- and Pik-mediated resistance (Cesari et al., 2014; Kanzaki et al., 2012), indirect recognition of the AVR-Pii effector by Pii has been proposed (Fujisaki et al., 2015). By using MutMap-Gap, a whole genome sequencing-based method of mutation identification, we have isolated an $N L R$ gene (Pii). In this study, we searched rice genes involved in Pii-mediated resistance, and identified another NLR gene required for Pii-mediated resistance. This new NLR gene as designated as Pii-2 is different from the previously-identified Pii gene (Takagi et al., 2013), which is here renamed to Pii-1, but is genetically linked to the Pii-1 locus. 


\section{RESULTS AND DISCUSSION}

\section{Screen of rice mutants showing deficiency of Pii-mediated resistance}

The rice cultivar Hitomebore is known to have Pii gene and shows resistance to rice blast fungus carrying AVR-Pii (Yoshida et al., 2009). In the previous study, we isolated Hitomebore ethylmethanesulfonate (EMS)-induced mutants (Hit5948 and Hit6780) showing susceptibility to an incompatible isolate of M. oryzae with AVR-Pii (TH68-126). These mutants had loss-of-function mutations in the same NLR gene and we concluded it is Pii (Takagi et al., 2013). In the current study, to identify other genes required for Pii-mediated resistance, we newly screened a total of 5,600 Hitomebore EMS mutant lines by spray inoculation with TH68-126, resulting in the isolation of five mutant lines (Hit5882, Hit12904, Hit13537, Hit13701 and Hit14409) showing susceptibility to TH68-126. In these mutant lines, genomic sequence of the previously identified Pii gene (Takagi et al., 2013) was determined by Sanger sequencing. Three of the five mutant lines had mutations in the Pii gene (Hit12904: exon-intron junction; Hit13537: Q540stop; and Hit14409: exon-intron junction), suggesting that these mutations contributed to their susceptibility to TH68-126. However, other two mutants, Hit5882 and Hit13701, had no mutation in the Pii gene. The susceptible phenotypes of Hit5882 and Hit13701 to the incompatible isolate TH68-126 were confirmed by punch inoculation test (Fig. 1 and S1).

\section{Identification of Pii-2 by MutMap-Gap}


To identify the causative mutations of Hit5882 and Hit13701, we carried out MutMap-Gap (Fig. S2). First, the genomic regions harboring candidate mutations of the two mutants were identified by MutMap analysis (Abe et al., 2012). To apply MutMap, Hit5882 and Hit13701 were separately crossed with the wild type Hitomebore (HitWT) to generate the F2 progeny (hereafter designated as Hit5882 F2 family and Hit13701 F2 family, respectively). Phenotype of each F2 progeny was studied by punch inoculation with an incompatible $M$. oryzae isolate TH68-126 carrying AVR-Pii. The F2 progeny of Hit5882 family segregated to resistance $(\mathrm{R})$ : susceptible $(\mathrm{S})$ in the ratio of $86: 33$ and that of Hit13701 family segregated to $\mathrm{R}: \mathrm{S}$ in the ratio of $83: 22$, both conforming to the $3: 1$ ratio (Chi square test; Hit5882 family: $\chi^{2}=0.47$, ns; Hit13701 family: $\chi^{2}=0.92, \mathrm{~ns}$ ), indicating that the susceptible phenotypes of both mutants were caused by single recessive mutations. For each family, DNA from all the susceptible F2 progeny (33 and 22 individuals for Hit5882 and Hit13701 family, respectively) were extracted and bulked in equal amount and subjected to whole genome sequencing by illumina NextSeq500 sequencer, resulting in 5.81 and $6.73 \mathrm{~Gb}$ sequence reads for Hit5882 and Hit13701 family, respectively (Table S1). The sequence reads were aligned by BWA software (Li et al., 2009) to "Hitomebore reference sequence", developed by replacing the nucleotides of publically available Nipponbare reference genome (Os-Nipponbare-Reference-IRGSP-1.0) with those of Hitomebore at all SNP positions between the two cultivars. The SNP-index values (Abe et al., 2012), representing the allele frequency of mutant nucleotides among the F2 pool, were calculated at all SNP positions using MutMap pipeline ver 1.4.4 (http://genome-e.ibrc.or.jp/home/bioinformatics-team/mutmap). MutMap analysis of the 
two families showed single peaks in the genomic region from 9.5 to $13 \mathrm{Mb}$ on Chromosome 9 (Fig. 2 and Fig. S3), which coincided with the previously-identified Pii gene (Takagi et al., 2013). None of the SNPs with SNP-index $=1$ caused amino acid changes or corresponded to splicing junctions of the putative genes located in the region. Since the MutMap analysis was carried out on the "Hitomebore reference sequence" developed from the Nipponbare reference genome by nucleotide replacements, the SNPs present in Hitomebore-specific genomic region were not addressed in this analysis. Our failure of identification of candidate mutations in the region suggests that the causative mutation may be located on such Hitomebore-specific genomic region that are missing from the "Hitomebore reference sequence".

To search for the mutations localized in Hitomebore specific genomic regions, we carried out local de novo assembly to reconstruct Hitomebore specific sequences corresponding to the Nipponbare 9.5-13 Mb genomic region on Chromosome 9. To this end, the Hitomebore sequence reads mapped to 9.5-13 Mb on Chromosome 9 (total 168.54 $\mathrm{Mb}$ in size) and those unmapped to "Hitomebore reference sequence" (total 688.19 Mb in size) were extracted, and were together subjected to de novo assembly using DISCOVAR de novo (Weisenfeld et al., 2014). This de novo assembly resulted in the generation of 2,087 contigs amounting to the total size of $6,234 \mathrm{~Kb}$ with N50 value of 3,431 bp (Fig. S4). Combined with "Hitomebore reference sequence", the sequences of the 2,087 contigs served as a new reference sequence. We aligned the bulked DNAs of mutant F2 of the Hit5882 family and Hit13701 family, respectively, to the new reference sequence. 
MutMap-Gap analyses of Hit5882 and Hit13701 using the new reference sequence identified candidate mutations (SNP-index=1) in the same contig "no.298" that was generated by de novo assembly (Table S2), which harbored a sequence corresponding to a truncated gene encoding a putative NLR protein. Since the gene in contig "no.298" was partial, the 5' and 3' termini of the cDNA sequence were determined by Rapid Amplification of c-DNA Ends (RACE) method. Based on the sequence information of both the 5' -and 3' -UTR regions, the full-length protein coding region was amplified by PCR, and the sequence was determined by Sanger method (Fig. S5). Next, to determine the genomic sequence of the NLR gene, the obtained cDNA sequence was subjected to BLAST search using a database of the contigs developed by de novo assembly. The BLAST search revealed that contig 298 and contig 1164 showed similarity to the region $1-2,581 \mathrm{bp}$ and 3,256-3,717 bp of the $N L R$ coding sequence, respectively, indicating that contig 298 and contig 1164 encode the upstream and downstream regions of the NLR genomic DNA, respectively. On the other hand, no contigs containing the internal region $(2,582-3,255 \mathrm{bp})$ of the NLR coding sequence was recovered by BLAST. We therefore PCR amplified this region and sequenced by Sanger method. The resulting 5'-, 3'- and internal regions of the gene were assembled and finally 7,946 bp genomic DNA carrying the $N L R$ gene of Hitomebore cultivar was determined (Fig. S5). The candidate mutation in Hit5882 causes an amino acid substitution (Val to Asp: V371D) and that in Hit13701 causes a nonsense mutation (Q247stop) in the NLR gene (Fig. 3 and Table S2).

To verify whether the disruption of this NLR gene in the contig "no.298" is responsible for the loss of Pii-mediated resistance in the two independent Hitomebore 
mutant lines, Hit5882 and Hit13701, we carried out an allelism test using F1 plants derived from a cross of Hit5882 and Hit13701. When the F1 plants derived from Hit5882 × Hit13701 were challenged with M. oryzae isolate TH68-126 with AVR-Pii, all three F1 plants showed the susceptible phenotype (Fig. 3). This result strongly suggests that the NLR gene in contig no.298 is required for the Pii-mediated resistance in Hitomebore. Since this NLR gene is located close to the previously-identified Pii gene (Fig. 2; Takagi et al., 2013), we named the newly identified gene as Pii-2. After confirming the sequence by Sanger sequencing, we registered this new NLR gene in NCBI (accession no LC190730) (Fig. S5). Accordingly, the gene defined as Pii in the previous study (Takagi et al., 2013) was renamed as Pii-1 (accession no AB820896).

The Pii-2 mutations in Hit5882 and Hit13701 are located at nucleotide-binding site (NBS) (Fig. S5). Complete loss of function of Pii-2 is expected by the nonsense mutation in Hit13701. On the other hand, the mutation in Hit5882 causes an amino acid substitution (V371D). Although this position is distant from the P-loop of NBS required for NLR function (Mestre and Baulcombe, 2006), the drastic effect of V371D mutation (Figs. 1 and S1) indicates the importance of V371 for Pii-2 activity.

A BLAST search revealed that Pii-1 and Pii-2 in Hitomebore showed a high amino acid sequence similarity with Pi5-1 and Pi5-2 (Lee et al., 2009), respectively, among the known rice $N L R$ genes (Fig. S6), indicating that Pii is allelic to Pi5. Between Pii and Pi5, we see some amino acid differences (Fig. S6). Currently, we do not know their importance in recognition specificity to different $A V R$ alleles. The ratio of nonsynonymous substitutions to synonymous substitutions (dN/dS) between Pii-1 vs Pi5-1 and Pii-2 vs 
Pi5-2 were 2.87 and 2.34, respectively. Higher levels of nonsynonymous substitutions as compared to synonymous substitutions implies that diversion between Pii and Pi5 may have involved positive selection.

Successful isolation of Pii-1 and Pii-2 by MutMap-Gap has demonstrated that MutMap-Gap enables identifying the genes that are absent from publicly available reference genome sequences (e.g. Nipponbare reference genome in rice). Genome organization around the rapidly-evolving genes including $N L R$ s are known to be highly variable (Yang et al., 2013). Therefore, MutMap-Gap may be especially useful to identify $N L R$ genes required for plant immunity. In this study, local de novo assembly for MutMap-Gap was carried out by DISCOVAR de novo using paired end sequence reads with 250 bp overlaps. This local de novo assembly achieved complete assembly of Pii-1 gene in the contig "no.51" while Pii-2 sequence was partial. Recent advance in PacBio sequencer (Au et al., 2012) will permit to generate much longer sequence reads than illumina sequencer, which may allow improvement of local de novo assembly. In future study we need to address the amount and length of sequence reads needed to perform high accuracy local de novo assembly by MutMap-Gap to apply to other crops with lager genome sizes.

In this study, we showed genetic evidence that not only Pii-1 but also Pii-2 is an essential component in Pii-mediated resistance. Resulting Pii-2 deficient mutants (Hit5882 and Hit13701) together with Pii-1 deficient mutants (Hit5948 and Hit6780) will enable us to perform experiments to analyze functions of Pii-1 and Pii-2. 


\section{EXPERIMENTAL PROCEDURES}

\section{Mutant screening}

A total 5,600 Hitomebore EMS mutant lines were applied to spray inoculation test with the incompatible rice blast (M. oryzae; isolate TH68-126) carrying AVR-Pii, as described in Takagi et al. (2013). The mutants screened by spray inoculation test were confirmed their phenotype by paunch inoculation test using the compatible isolate Sasa2 (AVR-Pii-) and the incompatible isolate Sasa2-AVRPii $\left(A V R-P i i^{+}\right)$which is generated by transformation of Sasa2 with AVR-Pii (Yoshida et al., 2009).

\section{MutMap-Gap analysis for identifying candidate gene}

MutMap-Gap analysis was carried out as described in Takagi et al. (2013). First, the genomic region harboring a candidate mutation was identified by the conventional MutMap method (Abe et al., 2012). Namely, the identified mutants were crossed with HitWT to generate the F2 segregating population. The F2 progeny were tested for their phenotypes by punch inoculation with an incompatible $M$. oryzae isolate. The DNAs obtained from the susceptible F2 individuals were bulked in equal amount and applied to library construction for illumina sequencer using TruSeq DNA PCR-Free LT Sample Prep Kit (illumina). The libraries were subjected to whole genome sequence by illumina sequencer NextSeq500. The MutMap analysis is carried out by pipeline version 1.4.4 (http://genome-e.ibrc.or.jp/home/bioinformatics-team/mutmap). In this MutMap analysis, the SNP positions where HitWT sequence shows low read depth $(<=5)$ were excluded. 
Following MutMap analysis, local de novo assembly was carried out to reconstruct Hitomebore specific genome corresponding to the candidate genomic region. For local de novo assembly, the sequence reads mapped to the candidate region and those unmapped were combined and subjected to assemble by DISCOVAR de novo (Weisenfeld et al., 2014). Finally, the sequence reads from the bulked DNA obtained from the F2 progeny showing mutant phenotype were aligned to the assembled contigs and SNP-index was calculated by MutMap pipeline. A flow chart of the procedure is given in Fig. S2.

To determine the full-length coding sequence of Pii-2 gene, the 5'- and 3'- terminal sequences of Pii-2 cDNA of the rice cultivar Hitomebore were determined by SMARTer ${ }^{\circledR}$ RACE cDNA Amplification Kit (Takara) using the gene specific primers 5'-GCAGACCTTTCCGGAGTGCTGATTGTCC-3' (for 5'- RACE) and 5'-AACAGCAGCCTCACTGAAATTGCGAAGC-3' (for 3'-RACE). Based on the sequence information of the 5'- and 3'- UTRs of Pii-2 cDNA, a cDNA fragment containing the full-length coding region of Pii-2 gene was amplified by RT-PCR using a primer set 5'-ATCCAGCGATACAATACCAGAAGT-3' and 5'-GCTGTGCCCAACCCCCATTTCAAT-3'. Resulting cDNA was cloned using Zero Blunt TOPO PCR cloning kit (Thermo Fisher Scientific) and the DNA sequence was determined by Sanger sequencing.

To determine the internal sequence of Pii-2 gene between contig 298 and contig 1164 (corresponding to the $2,582-3,255$ bp region of Pii-2 cDNA coding sequence), PCR was carried out using genomic DNA as template and the primers 5'-

CGATGGCTTCTCAGGTTGG -3' (corresponding to the internal region of Pii-2 cDNA) 
and 5'- TCCGGCACTGGTTTTGTG -3' (corresponding to contig 1164), and the resulting PCR product was subjected to Sanger sequencing (fragment 1). Based on the obtained sequence information, additional genomic PCR using the primers, 5'-

CCTCTCTATCACAAAACAAAACGAC -3' (corresponding to contig 298 sequence) and 5'- TGGCAAACAGCAGAGCATG -3' (corresponding to the internal genomic region of Pii-2) was performed, and the resulting PCR product was sequenced (fragment 2). DNA sequences of fragments 1 and 2 were assembled and used for reconstruction of the entire genomic sequence of the Pii-2 gene. 


\section{ACKNOWLEDGEMENTS}

We thank Dr Kentaro Yoshida (Kobe university) and members of our lab for discussions

and general assistance. This work was supported by Science and Technology Research

Promotion Program for Agriculture, Forestry, Fisheries and Food Industry, Japan,

Grant-in-aid for MEXT (Scientific Research on Innovative Areas 23113009) and JSPS

KAKENHI (Grant No. 26850029) and JSPS Kakenhi 15H05779. 


\section{FIGURE LEGENDS}

\section{Fig. 1}

\section{Rice Hitomebore mutants Hit5882 and Hit13701 deficient in Pii-mediated resistance.}

Rice leaf blade punch inoculation of M. oryzae isolate, TH68-126 (AVR-Pii+), to wild-type Hitomebore (HitWT : Pii+) and its mutants (Hit5882 and Hit13701). Inoculated leaves were photographed at 10 days after inoculation. HitWT shows resistance to TH68-126, whereas the two Hitomebore mutants, Hit5882 and Hit13701 are susceptible.

\section{Fig. 2}

\section{MutMap analysis of the mutant Hit5882 and Hit13701.}

(A) Segregation of wild-type and mutant phenotypes in the F2 families derived from a cross of the wild-type Hitomebore (HitWT) with Hit5882 (left) and that of HitWT with Hit13701 (right). Phenotype of F2 individuals was confirmed by punch inoculation of $M$. oryzae isolate TH68-126 carrying AVR-Pii. The numbers of F2 individuals showing wild-type (resistant) and mutant (susceptible) phenotypes are indicated at the bottom. (B) SNP-index plots of chromosome 9 in MutMap analysis for the mutations of Hit5882 and Hit13701. SNP-index for SNPs are given in blue dots. The red line indicates the sliding window average of SNP-index values of SNPs in $4 \mathrm{Mb}$ interval with $10 \mathrm{~kb}$ increment. Green line represents the $95 \%$ statistical confidence limit under the null hypothesis of SNP-index $=$ 0.5 . 


\section{Fig. 3}

\section{Identification of causative mutations for Hit5882 and Hit13701 by MutMap-Gap.}

(A) Gene structure of Pii-2 and the SNP positions identified in Hit5882 (1371) and Hit13701 (998). Purple and red box indicates the untranslated region (UTR) and exon, respectively. Introns are indicated by horizontal bar. (B) Allelism test for Hit5882 and Hit13701 mutations. The F1 plants obtained from a cross between Hit5882 and Hit13701 were subjected to the punch inoculation with M. oryzae isolate TH68-126 carrying AVR-Pii. Heterozygosity of the two mutations of Hit5882 and Hit13701 in the F1 plants were confirmed by Sanger sequence. Arrowheads indicated the candidate mutations for Hit5882 and Hit13701. All F1 plants (Hit5882/Hit13701) showed susceptibility, suggesting that the mutations in the newly identified NLR gene is responsible for the Hit5882 and Hit13701 phenotypes. 


\section{REFERENCES}

Abe, A., Kosugi, S., Yoshida, K., Natsume, S., Takagi, H., Kanzaki, H., Matsumura, H., Yoshida, K., Mitsuoka, C., Tamiru, M., Innan, H., Cano, L., Kamoun, S. and Terauchi, R. (2012) Genome sequencing reveals agronomically important loci in rice using MutMap. Nat. Biotechnol. 30, 174-178.

Ashikawa, I., Hayashi, N., Abe, F., Wu, J. and Matsumoto, T. (2012) Characterization of the rice blast resistance gene Pik cloned from Kanto51. Mol. Breed. 30, 485-494.

Au, K.F., Underwood, J.G., Lee, L. and Wong, W.H. (2012) Improving PacBio long read accuracy by short read alignment. PLoS One 7, e46679.

Cesari, S., Thilliez, G., Ribot, C., Chalvon, V., Michel, C., Jauneau, A., Rivas, S., Alaux, L., Kanzaki, H., Okuyama, Y., Morel, J.B., Fournier, E., Tharreau, D., Terauchi, R. and Kroj, T. (2013) The rice resistance protein pair RGA4/RGA5 recognizes the Magnaporthe oryzae effectors AVR-Pia and AVR1-CO39 by direct binding. Plant Cell 25, 1463-1481.

Cesari, S., Bernoux, M., Moncuquet, P., Kroj, T. and Dodds, P. N. (2014) A novel conserved mechanism for plant NLR protein pairs: the 'integrated decoy'hypothesis. Front Plant Sci 5, 606.

Dean, R., Van Kan, J.A., Pretorius, Z.A., Hammond-Kosack, K.E., Di Pietro, A., Spanu, P.D., Rudd, J.J., Dickman, M., Kahmann, R., Ellis, J. and Foster, G. D. (2012) The Top 10 fungal pathogens in molecular plant pathology. Mol. Plant Pathol. 13, 414-430. 
Duxbury, Z., Ma, Y., Furzer, O.J., Huh, S.U., Cevik, V., Jones, J.D. and Sarris, P.F. (2016) Pathogen perception by NLRs in plants and animals: parallel worlds. Bioessays 38, 769-781.

Eitas, T.K., and Dangl, J.L. (2010) NB-LRR proteins: pairs, pieces, perception, partners, and pathways. Curr. Opin. Plant Biol. 13, 472-477.

Flor, H.H. (1971) Current status of the gene-for-gene concept. Annu Rev Phytopathol 9, $275-296$.

Fujisaki, K., Abe, Y., Ito, A., Saitoh, H., Yoshida, K., Kanzaki, H., Kanzaki, E., Utsushi, H., Yamashita, T., Kamoun, S. and Terauchi, R. (2015) Rice Exo70 interacts with a fungal effector, AVR-Pii, and is required for AVR-Pii-triggered immunity. Plant J. 83, 875-887.

Ishizaki, K., Hoshi, T., Abe, S.I., Sasaki, Y., Kobayashi, K., Kasaneyama, H., Matsui, T.A. and Azuma, S. (2005) Breeding of blast resistant isogenic lines in rice variety" Koshihikari" and evaluation of their characters. Breeding science 55, 371-377.

Kanzaki, H., Yoshida, K., Saitoh, H., Fujisaki, K., Hirabuchi, A., Alaux, L., Fournier, E., Tharreau, D. and Terauchi, R. (2012) Arms race co-evolution of Magnaporthe oryzae AVR-Pik and rice Pik genes driven by their physical interactions. Plant J. 72, 894-907.

Li, H. and Durbin, R. (2009) Fast and accurate short read alignment with BurrowsWheeler transform. Bioinformatics 25, 1754-1760.

Mestre, P. and Baulcombe, D.C. (2006) Elicitor-mediated oligomerization of the tobacco $\mathrm{N}$ disease resistance protein. Plant Cell 18, 491-501. 
Miah, G., Rafii, M.Y., Ismail, M.R., Puteh, A.B., Rahim, H.A., Asfaliza, R. and Latif, M.A. (2013) Blast resistance in rice: a review of conventional breeding to molecular approaches. Mol. Biol. Rep. 40, 2369-2388.

Nishimura, M.T. and Dangl, J.L. (2014) Paired plant immune receptors. Science 344, 267-268.

Okuyama, Y., Kanzaki, H., Abe, A., Yoshida, K., Tamiru, M., Saitoh, H., Undan, J. Ito, A., Sone, T. and Terauchi, R. (2011) A multifaceted genomics approach allows the isolation of the rice Pia-blast resistance gene consisting of two adjacent NBSLRR protein genes. Plant J. 66, 467-479.

Takagi, H., Uemura, A., Yaegashi, H., Tamiru, M., Abe, A., Mitsuoka, C., Saitoh, H., Utsushi, H., Natsume, S., Kanzaki, H., Matsumura, H., Saitoh, H., Yoshida, K., Cano, L.M., Kamoun, S. and Terauchi R. (2013) MutMap-Gap: whole-genome resequencing of mutant F2 progeny bulk combined with de novo assembly of gap regions identifies the rice blast resistance gene Pii. New Phytol. 200, 276-283.

Win, J., Chaparro-Garcia, A., Belhaj, K., Saunders, D. G. O., Yoshida, K., Dong, S., Schornack, S, Zipfel, C., Robatzek, S., Hogenhout, S.A. and Kamoun, S. (2012) Effector biology of plant-associated organisms: concepts and perspectives. In Cold Spring Harbor symposia on quantitative biology (Vol. 77, pp. 235-247). Cold Spring Harbor Laboratory Press.

Weisenfeld, N.I., Yin, S., Sharpe, T., Lau, B., Hegarty, R., Holmes, L., Sogoloff, B., Tabbaa, D., Williams, L., Russ, C., Nusbaum, C., Lander, E.S., MacCallum, I. 
and Nusbaum, C. (2014) Comprehensive variation discovery in single human genomes. Nat. Genet. 46, 1350-1355.

Wu, C.H., Abd-El-Haliem, A., Bozkurt, T.O., Belhaj, K., Terauchi, R., Vossen, J.H. and Kamoun, S. (2017) NLR network mediateds immunity to diverse plant pathogens. Proc. Natl. Acad. Sci. U.S.A. 114, 8113-8118.

Yang, S., Li, J., Zhang, X., Zhang, Q., Huang, J., Chen, J.Q., Hartl, D.L. and Tian, D. (2013) Rapidly evolving R genes in diverse grass species confer resistance to rice blast disease. Proc. Natl. Acad. Sci. U.S.A. 110, 18572-18577.

Yoshida, K., Saitoh, H., Fujisawa, S., Kanzaki, H., Matsumura, H., Yoshida, K., Tosa, Y., Chuma, I., Takano, Y., Win, J., Kamoun, S. and Terauchi, R. (2009) Association genetics reveals three novel avirulence genes from the rice blast fungal pathogen Magnaporthe oryzae. Plant Cell 21, 1573-1591.

Yuan, B., Zhai, C., Wang, W., Zeng, X., Xu, X., Hu, H., Lin, F., Wang, L. and Pan, Q. (2011) The Pik-p resistance to Magnaporthe oryzae in rice is mediated by a pair of closely linked CC-NBS-LRR genes. Theor. Appl. Genet. 122, 1017-1028.

Zeigler, R.S., Leong, S.A., and Teng, P.S. (1994) Rice blast disease. Int. Rice Res. Inst.

Zhai, C., Lin, F., Dong, Z., He, X., Yuan, B., Zeng, X., Ling, W. and Pan, Q. (2011)

The isolation and characterization of $P i k$, a rice blast resistance gene which emerged after rice domestication. New Phytol. 189, 321-334. 
bioRxiv preprint doi: https://doi.org/10.1101/227132; this version posted November 30, 2017. The copyright holder for this preprint (which was not certified by peer review) is the author/funder. All rights reserved. No reuse allowed without permission.

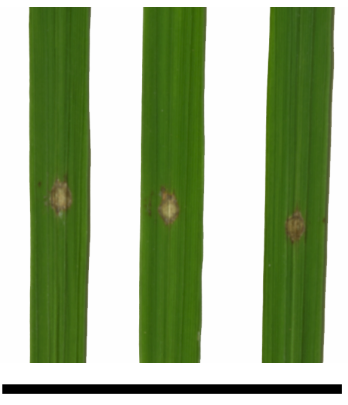

HitWT

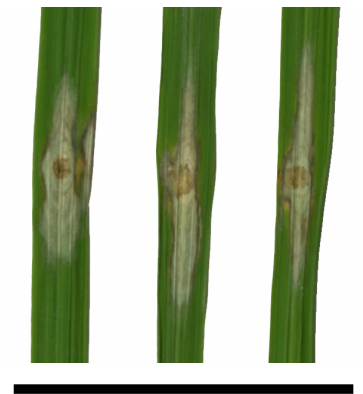

Hit5882

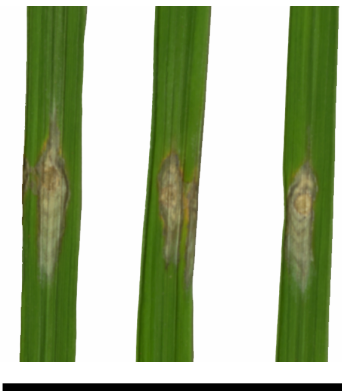

Hit13701 
A
Wild type

86

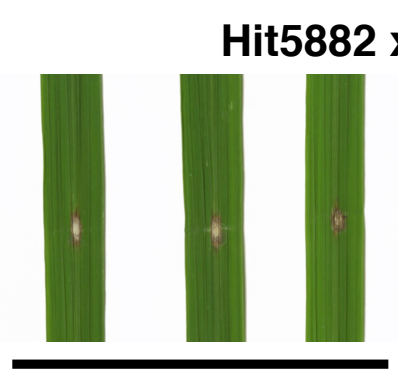

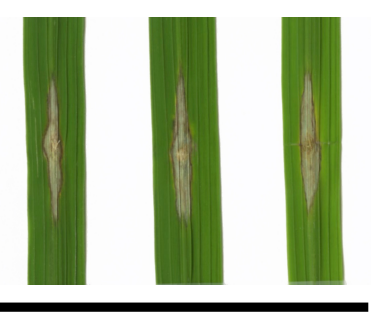

Mutant type

33

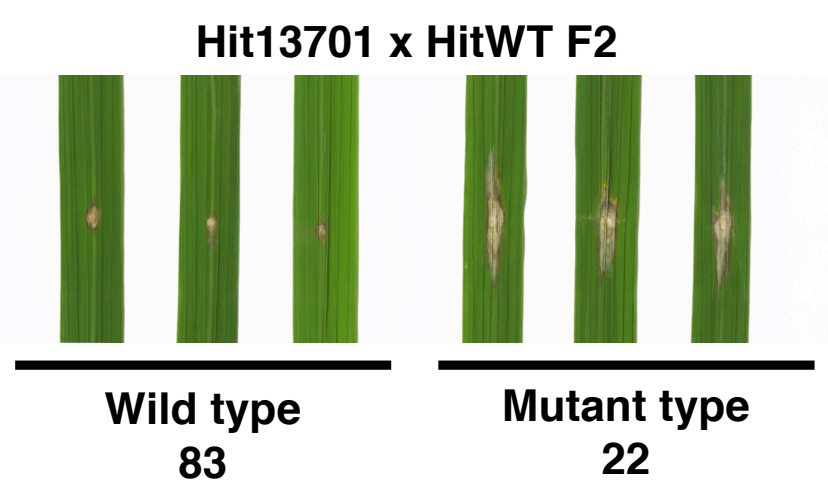

B Hit5882 x HitWT F2 bulk Chromosome 9

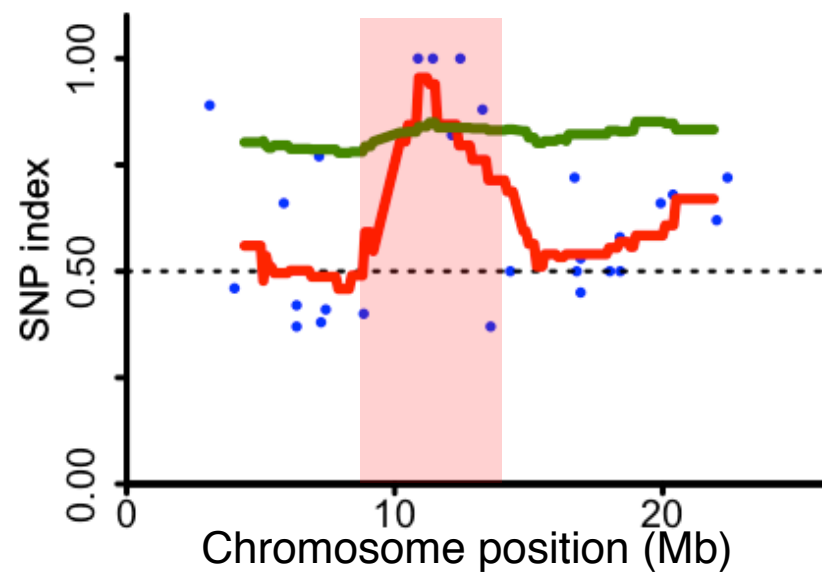

Hit13701 x HitWT F2

Hit13701 x HitWT F2 bulk Chromosome 9

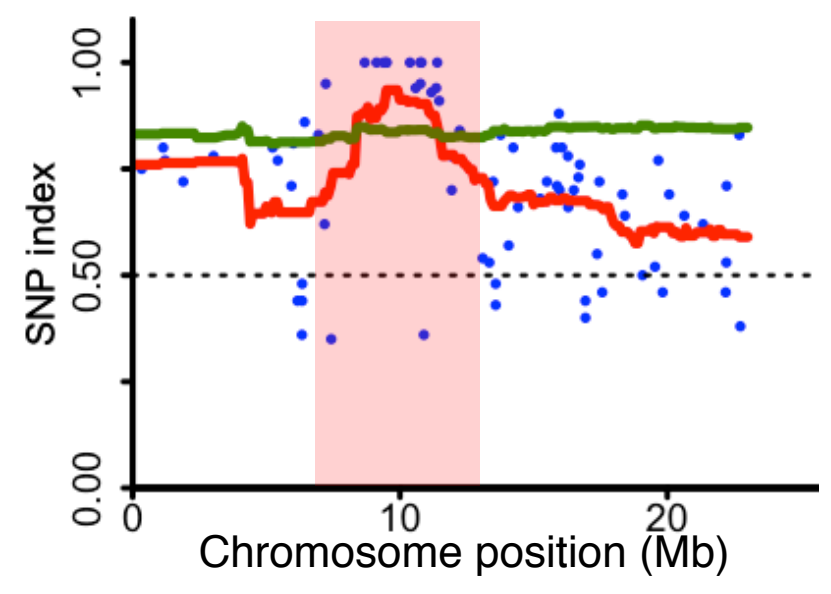




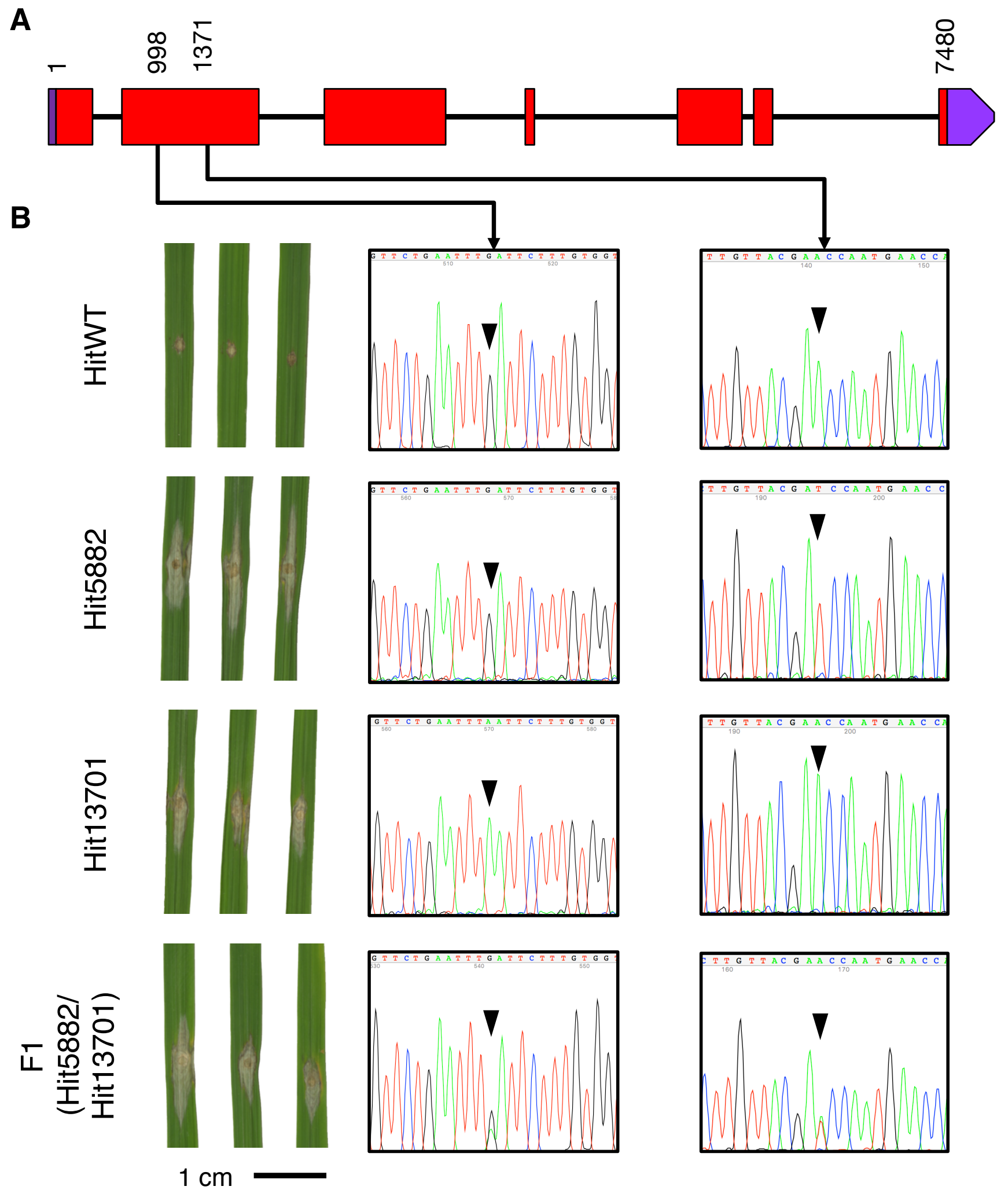

